\title{
Recipe for ultrafast and persistent phase-change memory materials
}

\author{
Keyuan Ding ${ }^{1}$, Bin Chen ${ }^{1,2}$, Yimin Chen ${ }^{3,4}$, Junqiang Wang ${ }^{5}$, Xiang Shen ${ }^{4}$ and Feng Rao ${ }^{1}$
}

\begin{abstract}
The contradictory nature of increasing the crystallization speed while extending the amorphous stability for phasechange materials (PCMs) has long been the bottleneck in pursuing ultrafast yet persistent phase-change randomaccess memory. Scandium antimony telluride alloy $\left(\mathrm{Sc}_{x} \mathrm{Sb}_{2} \mathrm{Te}_{3}\right)$ represents a feasible route to resolve this issue, as it allows a subnanosecond SET speed but years of reliable retention of the RESET state. To achieve the best device performances, the optimal composition and its underlying working mechanism need to be unraveled. Here, by tuning the doping dose of $\mathrm{Sc}$, we demonstrate that $\mathrm{Sc}_{0.3} \mathrm{Sb}_{2} \mathrm{Te}_{3}$ has the fastest crystallization speed and fairly improved data nonvolatility. The simultaneous improvement in such 'conflicting' features stems from reconciling two dynamics factors. First, promoting heterogeneous nucleation at elevated temperatures requires a higher Sc dose to stabilize more precursors, which also helps suppress atomic diffusion near ambient temperatures to ensure a rather stable amorphous phase. Second, however, enlarging the kinetic contrast through a fragile-to-strong crossover in the supercooled liquid regime should require a moderate Sc content; otherwise, the atomic mobility for crystal growth at elevated temperatures will be considerably suppressed. Our work thus reveals the recipe by tailoring the crystallization kinetics to design superior PCMs for the development of high-performance phase-change working memory technology.
\end{abstract}

\section{Introduction}

Commercialized phase-change random-access memory (PCRAM) stores digital information in the amorphous and crystalline phases of chalcogenide phase-change materials (PCMs), such as $\mathrm{Ge}_{2} \mathrm{Sb}_{2} \mathrm{Te}_{5}{ }^{1-3}$. Reversible switching between these two phases of $\mathrm{Ge}_{2} \mathrm{Sb}_{2} \mathrm{Te}_{5}$ in a PCRAM device is typically induced by fast electrical pulse (Joule) heating for tens of nanoseconds ${ }^{4}$. The encoded data, in the power-off state, can be reliably stored for tens of years at ambient temperatures ${ }^{5}$. Such swift and nonvolatile features, as well as the merits ${ }^{4,6}$ of high scalability, low power consumption, and long cycling endurance,

\footnotetext{
Correspondence: Feng Rao (fengrao@szu.edu.cn)

${ }^{1}$ College of Materials Science and Engineering, Shenzhen University, 518060 Shenzhen, China

${ }^{2}$ Key Laboratory of Optoelectronic Devices and Systems of Ministry of Education and Guangdong Province, College of Optoelectronic Engineering, Shenzhen University, 518060 Shenzhen, China

Full list of author information is available at the end of the article

These authors contributed equally: Keyuan Ding, Bin Chen, Yimin Chen
}

make PCRAM the best candidate for realizing an ideal "universal memory" to renovate the current computing system based on the classic von Neumann architecture ${ }^{7}$. Since 2015 until very recently, Intel's Optane $D^{8}$ and Micron's X100 NVMe (https://www.micron.com/ products/advanced-solutions/3d-xpoint-technology/ x100) chips, both employing 3D Xpoint PCRAM technology, served as storage-class memory to mitigate the widening performance gap (memory wall) between volatile dynamic random-access memory (DRAM) and nonvolatile solid-state drive flash memory. Nevertheless, substitution of traditional working memories, i.e., static random-access memory and DRAM, has long been recognized as impossible ${ }^{7}$ owing to the stringent requirements of even faster (sub $\sim 1-10 \mathrm{~ns}$ ) operating speeds and years of data nonvolatility for PCRAM devices $^{9-11}$.

Such a speed bottleneck of the PCRAM devices originates from the relatively sluggish crystallization process in 
the SET operation, not from the amorphization procedure for the RESET operation ${ }^{2,12}$. Generally, the whole crystallization process consists of two stages: the nucleation of small crystallites and their subsequent growth. Note that the crystal growth velocity in the supercooled liquid of $\mathrm{Ge}_{2} \mathrm{Sb}_{2} \mathrm{Te}_{5}$ can be rather fast, reaching a maximum of $\sim 0.5-3.0 \mathrm{~m} \mathrm{~s}^{-1}$ at $\sim 600-700 \mathrm{~K}^{13-15}$. In a relatively miniaturized PCRAM device of $\sim 7.5 \times 17.0 \mathrm{~nm}^{2}$ in cross section, even when using $\mathrm{Sb}$-rich $\mathrm{Ge}_{2} \mathrm{Sb}_{2} \mathrm{Te}_{5}$ to further strengthen the growth momentum, the shortest SET operation still takes $\sim 30 \mathrm{~ns}^{16}$. Such a 'limited' speed performance suggests that in addition to the strong (inward) growth momentum from peripheral crystalline interfaces, it would be beneficial to also have copious small crystallites inside the glassy matrix that can quickly grow simultaneously; the two processes would work together to facilitate an even faster crystallization ${ }^{9,10,17}$. However, the nucleation of $\mathrm{Ge}_{2} \mathrm{Sb}_{2} \mathrm{Te}_{5}$ faces an unavoidable 'time' issue, namely, it is stochastic in nature because the time for incubating stable nuclei over the critical size is distributed over a broad scale, from several hundreds of picoseconds to many nanoseconds ${ }^{9,18}$. The stochasticity is associated with the short lifetime of subcritical nuclei that consist of cubic motifs constructed by $\mathrm{Ge}(\mathrm{Sb})-\mathrm{Te}$ bonds ${ }^{11,12,19}$. These soft chemical bonds constantly form and rupture at high crystallization temperatures, e.g., $\sim 500-700 \mathrm{~K}$; therefore, these nucleation embryos fluctuate severely, surviving for only several picoseconds ${ }^{11}$. One may thus resort to a common solution by introducing stable heterogeneities into a glassy phase to elevate the nucleation rate. However, a difficulty arises here from the contradictory nature of $\mathrm{Ge}_{2} \mathrm{Sb}_{2} \mathrm{Te}_{5}$-like PCMs that (most of the) doping $^{20-22}$ always extends the stability of the amorphous phase for long-term data retention but inadvertently slows down the crystallization. The fast and persistent features of the phase-change working memory are seemingly irreconcilable, and a trade-off seems inevitable.

To solve this problem, we designed a $\mathrm{Sc}_{0.2} \mathrm{Sb}_{2} \mathrm{Te}_{3}$ alloy and validated its superior functions in a conventional PCRAM device, which enabled a 0.7-ns SET speed and a comparable data retention ability to the $\mathrm{Ge}_{2} \mathrm{Sb}_{2} \mathrm{Te}_{5}$ device of the same geometry ${ }^{11}$. The high-strength $\mathrm{Sc}-\mathrm{Te}$ bonds effectively enhance the stability of nucleation embryos, prolonging the lifetime against thermal fluctuations. Moreover, the embedded Sc-Te embryos are geometrically conformable with their crystalline counterpart, i.e., rocksalt $\mathrm{Sb}_{2} \mathrm{Te}_{3}$, lowering the surface free energy. These two traits together prompt a dramatic decrease in the energy barrier for nucleation, reducing the stochasticity and thus shortening the wait time for incubation ${ }^{11}$. We have also elucidated that Sc addition can create an enlarged kinetic contrast inside the supercooled liquid state over a fairly narrow temperature range ${ }^{23}$. This guarantees high atomic mobility at elevated temperatures for rapid crystal growth and suppressed atomic diffusion near room temperature for good data retention.

At this point, one may ask another question that is worth exploring: is there a better composition than $\mathrm{Sc}_{0.2} \mathrm{Sb}_{2} \mathrm{Te}_{3}$ to achieve an even faster SET speed and better data nonvolatility of the PCRAM device at the same time? In this work, we provide direct evidence to answer this question by systematically characterizing the structural, electrical, and thermal properties of a series of $\mathrm{Sc}_{\mathrm{x}} \mathrm{Sb}_{2} \mathrm{Te}_{3}$ compounds. We reveal that as the Sc content increases, the amorphous stability of $\mathrm{Sc}_{\mathrm{x}} \mathrm{Sb}_{2} \mathrm{Te}_{3}$ films can be enhanced monotonically, whereas balancing the nucleation rate with the growth velocity leads to the optimal Sc dose of $\mathrm{x} \sim 0.3$, which enables the fastest crystallization speed and improved data retention ability. Extra Sc doping restrains the kinetics substantially throughout the whole temperature range, reducing the crystallization speed. Our work may serve as a useful example demonstrating how to optimize the composition of PCMs through tailoring of their crystallization kinetics, aiming at the development of high-performance phasechange working- and storage-class memories.

\section{Materials and methods \\ Film preparation and characterization}

Approximately 300-nm-thick $\mathrm{Sc}_{x} \mathrm{Sb}_{2} \mathrm{Te}_{3}(x=0.1,0.2$, 0.3 , and 0.36) films were deposited on $\mathrm{SiO}_{2} / \mathrm{Si}$ substrates at room temperature by cosputtering pure $\mathrm{Sc}$ and $\mathrm{Sb}_{2} \mathrm{Te}_{3}$ targets in ultrahigh vacuum with a base pressure of $<\sim 2 \times$ $10^{-7}$ mTorr. The deposition rate was controlled to $\sim 5 \mathrm{~nm}$ $\min ^{-1} . G e_{2} \mathrm{Sb}_{2} \mathrm{Te}_{5}$ films of the same thickness were fabricated by sputtering the pure alloy target. The compositions of all the films were determined by using X-ray fluorescence spectroscopy (Rigaku RIX 2100). The sheet resistance of all the films was studied using a Linkam HFS600E-PB4 hot stage with a temperature accuracy of $\sim 0.1 \mathrm{~K}$. The morphology and crystallinity of $\sim 220^{\circ} \mathrm{C}$ annealed $\mathrm{Sc}_{x} \mathrm{Sb}_{2} \mathrm{Te}_{3}$ films ( $\sim 20 \mathrm{~nm}$ in thickness) were confirmed by high-resolution transmission electron microscopy (HRTEM, JEOL JEM-2100F) at a high tension of $200 \mathrm{kV}$. An $\sim 10$-nm-thick $\mathrm{SiO}_{2}$ capping layer was in situ grown on top of each film inside the vacuum chamber to avoid oxidation for HRTEM and thermal measurement.

\section{Device fabrication and electrical measurement}

Conventional T-shaped PCRAM devices with a tungsten bottom electrode contact (BEC) of $\sim 80-190 \mathrm{~nm}$ in diameter $(\phi)$ were fabricated using the $0.13 \mu \mathrm{m}$ node complementary metal-oxide semiconductor technology ${ }^{11}$. The thickness of the PCM films in all devices was controlled to $\sim 150 \mathrm{~nm}$. Approximately 15 -nm-thick TiN and $\sim 300$-nm-thick Al films were used as the top electrode in all devices. All electrical measurements were performed 

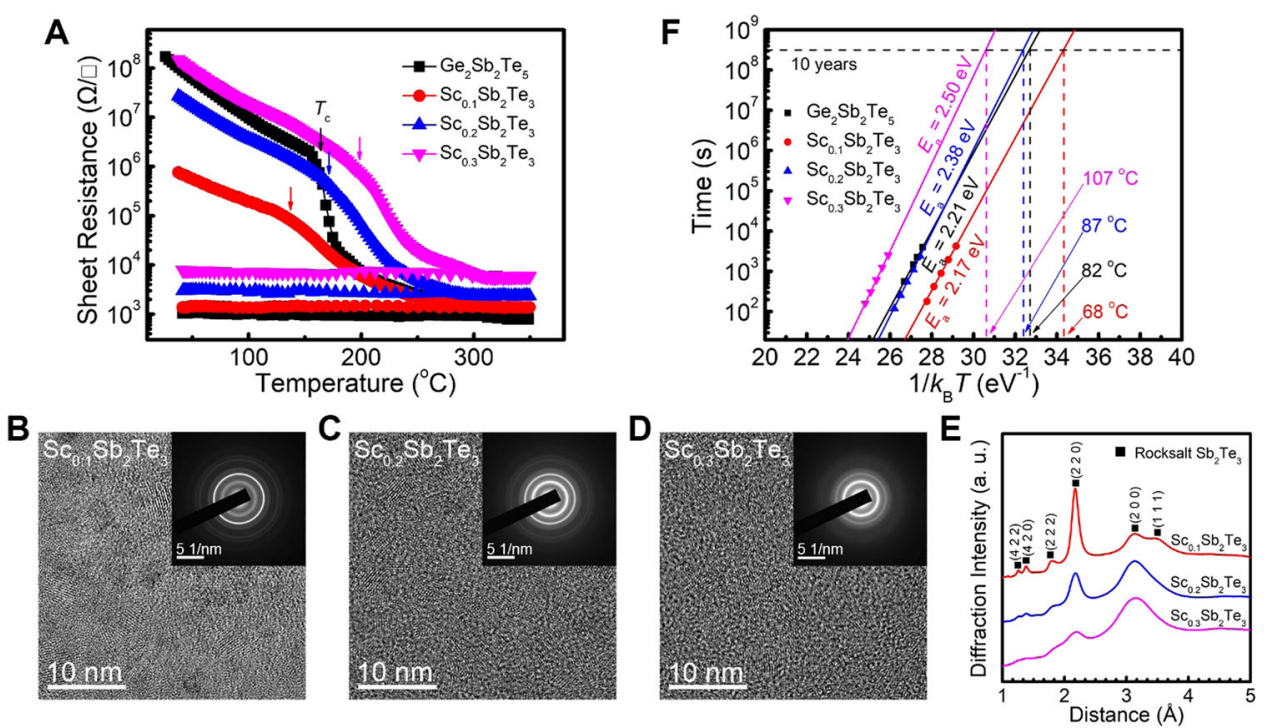

Fig. 1 Structural transformation and data retention ability. a Temperature dependence of the sheet resistance of $\sim 300-\mathrm{nm}_{\mathrm{n}}-\mathrm{thick} \mathrm{Ge}_{2} \mathrm{Sb}_{2} \mathrm{Te}_{5}$ and $\mathrm{Sc}_{x} \mathrm{Sb}_{2} \mathrm{Te}_{3}(x=0.1,0.2$, and 0.3$)$ films at the same heating rate of $10^{\circ} \mathrm{C} \mathrm{min}{ }^{-1}$. The crystallization temperature $\left(T_{c}\right)$ is indicated by the arrow: 138 , $\sim 170, \sim 199$, and $\sim 163^{\circ} \mathrm{C}$ for the $\mathrm{Sc}_{0.1} \mathrm{Sb}_{2} \mathrm{Te}_{3}, \mathrm{Sc}_{0.2} \mathrm{Sb}_{2} \mathrm{Te}_{3}, \mathrm{Sc}_{0.3} \mathrm{Sb}_{2} \mathrm{Te}_{3}$, and $\mathrm{Ge}_{2} \mathrm{Sb}_{2} \mathrm{Te}_{5}$ films, respectively. b-d BF-TEM images of $\sim 20$-nm-thick $\mathrm{SC}_{x} \mathrm{Sb}_{2} \mathrm{Te}_{3}$ films annealed at $\sim 220^{\circ} \mathrm{C}$. The SAED pattern of each sample is shown in the corresponding inset. e Raw radially integrated diffraction curves of the electron diffraction intensity extracted from the respective SAED patterns shown in (b-d). $\mathbf{f}$ Ten-year data retention abilities for $\mathrm{Ge}_{2} \mathrm{Sb}_{2} \mathrm{Te}_{5}$ and $\mathrm{Sc}_{x} \mathrm{Sb}_{2} \mathrm{Te}_{3}$ devices. The data were fitted using the Arrhenius equation $t=A \exp \left(E_{\mathrm{a}} / \mathrm{k}_{\mathrm{B}} T\right)$, where $t$ is the time to failure when the cell resistance in the RESET state, at a certain isothermal heating temperature, falls to half of its initial magnitude, $A$ is a proportionality constant, $E_{\mathrm{a}}$ is the activation energy, and $k_{B}$ is the Boltzmann constant.

by using a Keithley $2400 \mathrm{C}$ source meter (measuring the cell resistance), a Tektronix AWG5002B pulse generator (generating a voltage pulse with a minimum width of $\sim 6 \mathrm{~ns}$ ), a homemade constant current driver (generating a current pulse with a maximum magnitude of $\sim 10 \mathrm{~mA}$ ) and a Tektronix 7054 digital phosphor oscilloscope (measuring the transient voltage drop across the cell when the current pulse was applied).

\section{Flash differential scanning calorimetry (FDSC) measurements}

Power-compensation differential scanning calorimetry (DSC) was performed using a Mettler-Toledo FDSC 1 instrument with sensor chips (USF-1), each containing working and reference areas. $\mathrm{Sc}_{x} \mathrm{Sb}_{2} \mathrm{Te}_{3}$ small flakes were scraped off from the substrate and then transferred onto the working area of the chip sensor. The heating rate $(\Phi)$ was varied from 10 to $40,000 \mathrm{~K} \mathrm{~s}^{-1}$. At each $\Phi$, measurements were repeated at least three times for low $\Phi$ and 5-10 times for high $\Phi$, as the values of the crystallization temperature become more scattered at high $\Phi$. The thermal lag and temperature calibration of the FDSC are evaluated in detail in the Supplementary information. The detailed methodology of Kissinger fitting can be seen in our previous work ${ }^{24}$. The viscosity model used in this work is illustrated in the Supplementary information. Since the $\mathrm{Sc}_{x} \mathrm{Sb}_{2} \mathrm{Te}_{3}$ flakes were only one-side capped, it is appropriate to compare the FDSC results with those of uncapped $\mathrm{Ge}_{2} \mathrm{Sb}_{2} \mathrm{Te}_{5}$ films ${ }^{13,23}$.

\section{Density functional theory simulations}

Density functional theory (DFT) simulations were carried out with the Vienna ab initio Simulations Package $(\text { VASP })^{25}$. The Perdew-Burke-Ernzerhof functionals ${ }^{26}$ and the projector augmented wave pseudopotentials ${ }^{27}$ were used for VASP. Rocksalt $\mathrm{Sc}_{0.3} \mathrm{Sb}_{1.7} \mathrm{Te}_{3}$ supercells with 180 (11 Sc, $61 \mathrm{Sb}$, and $108 \mathrm{Te}$ ) atoms were simulated with periodic boundary conditions by NVT DFT-based molecular dynamics (DFMD). The model was heated from 300 to $1150 \mathrm{~K}$ at a heating rate of $15 \mathrm{~K} \mathrm{ps}^{-1}$. The energy cutoff was $180 \mathrm{eV}$, and the time step was $3 \mathrm{fs}$.

\section{Results and discussion}

\section{Structural transformation and stability of the amorphous} phase

Temperature-dependent electrical resistance measurements were first performed for as-deposited and $~ 300$ nm-thick $\mathrm{Ge}_{2} \mathrm{Sb}_{2} \mathrm{Te}_{5}$ and $\mathrm{Sc}_{x} \mathrm{Sb}_{2} \mathrm{Te}_{3}(x=0.1,0.2$, and 0.3) films upon in situ annealing at a heating rate of $10^{\circ} \mathrm{C}$ $\mathrm{min}^{-1}$ (Fig. 1a) since this technique is very sensitive to structural changes in $\mathrm{PCMs}^{28}$. All three as-deposited $\mathrm{Sc}_{\mathrm{x}} \mathrm{Sb}_{2} \mathrm{Te}_{3}$ films are in amorphous states, and the initial magnitude of the sheet resistance becomes larger as the Sc content $(\mathrm{x})$ increases from 0.1 to 0.3 , corresponding to the 
widened energy band gap of the amorphous semiconductor $^{28}$. When heated beyond the crystallization temperature $\left(T_{\mathrm{c}}\right)$ to $\sim 350^{\circ} \mathrm{C}$, the amorphous semiconductor finally transforms into a narrow-gap crystalline semiconductor of a stable crystalline (hexagonal) phase, with a much ( $\sim 3-4$ orders of magnitude) smaller sheet resistance.

We selected three annealed (at $\sim 220^{\circ} \mathrm{C}$ ) samples to assess the microstructural details by HRTEM. The brightfield (BF) HRTEM image and the corresponding selectedarea electron diffraction (SAED) pattern of each $\mathrm{Sc}_{x} \mathrm{Sb}_{2} \mathrm{Te}_{3}$ film show a homogeneous polycrystalline morphology with many nanosized crystal grains, indicating a nucleation-dominant crystallization behavior (Fig. $1 \mathrm{~b}-\mathrm{d})$. Figure $1 \mathrm{e}$ shows the raw radially integrated diffraction curves of the electron diffraction intensity extracted from the inset SAED patterns in Fig. 1b-d. All the diffraction peaks located in the range from $\sim 1$ to $\sim 4 \AA$ well match the featured positions of pure rocksalt $\mathrm{Sb}_{2} \mathrm{Te}_{3}{ }^{29}$, proving that all the annealed $\mathrm{Sc}_{x} \mathrm{Sb}_{2} \mathrm{Te}_{3}$ samples are of the rocksalt phase. As the Sc content increases, the diffraction intensity decreases, accompanied by peak broadening, correlating to smaller crystal grain sizes and poorer crystallinity. It is observable that some tiny crystallites are embedded in the amorphous networks of $\mathrm{Sc}_{0.2} \mathrm{Sb}_{2} \mathrm{Te}_{3}$ (Fig. 1c) and $\mathrm{Sc}_{0.3} \mathrm{Sb}_{2} \mathrm{Te}_{3}$ (Fig. 1d). Note that each $\mathrm{Sc}_{x} \mathrm{Sb}_{2} \mathrm{Te}_{3}$ film has a comparably wide temperature window (from $T_{\mathrm{c}}$ to $\sim T_{\mathrm{c}}+100^{\circ} \mathrm{C}$ ) to that of $\mathrm{Ge}_{2} \mathrm{Sb}_{2} \mathrm{Te}_{5}$ for retaining the metastable rocksalt phase ${ }^{11,29}$. This is beneficial for guaranteeing the amorphous-to-rocksalt transition for the SET operation of $\mathrm{Sc}_{x} \mathrm{Sb}_{2} \mathrm{Te}_{3}$ devices through the design of a suitable heating profile. Highspeed PCRAM application should avoid the involvement of a stable hexagonal phase because its formation requires a longer SET time, and its melting requires extra RESET energy $^{29}$.

Since the $\mathrm{Sc}_{0.2} \mathrm{Sb}_{2} \mathrm{Te}_{3}$ and $\mathrm{Ge}_{2} \mathrm{Sb}_{2} \mathrm{Te}_{5}$ films have close $T_{\mathrm{c}}$ values (Fig. 1a), the 10-year data retention ability of the RESET state for the $\mathrm{Sc}_{0.2} \mathrm{Sb}_{2} \mathrm{Te}_{3}$ device $\left(\sim 87^{\circ} \mathrm{C}\right)$ is slightly better than that of the $\mathrm{Ge}_{2} \mathrm{Sb}_{2} \mathrm{Te}_{5}$ device $\left(\sim 82^{\circ} \mathrm{C}\right)$, as shown in Fig. 1f. It is obvious that increasing (decreasing) the Sc content results in a higher (lower) $T_{\mathrm{c}}$ of the $\mathrm{Sc}_{0.3} \mathrm{Sb}_{2} \mathrm{Te}_{3}\left(\mathrm{Sc}_{0.1} \mathrm{Sb}_{2} \mathrm{Te}_{3}\right)$ film; correspondingly, the 10year data retention ability of the RESET state becomes superior $\left(\sim 107^{\circ} \mathrm{C}\right)$ and inferior $\left(\sim 68^{\circ} \mathrm{C}\right)$ for the $\mathrm{Sc}_{0.3} \mathrm{Sb}_{2} \mathrm{Te}_{3}$ and $\mathrm{Sc}_{0.1} \mathrm{Sb}_{2} \mathrm{Te}_{3}$ devices, respectively. The improved data retention ability should also be attributed to the increased crystallization activation energy $\left(E_{\mathrm{a}}\right)$ of the $\mathrm{Sc}_{0.2} \mathrm{Sb}_{2} \mathrm{Te}_{3}$ and $\mathrm{Sc}_{0.3} \mathrm{Sb}_{2} \mathrm{Te}_{3}$ devices, i.e., $\sim 2.38$ and $\sim 2.50 \mathrm{eV}$, respectively, compared to that $(\sim 2.21 \mathrm{eV})$ of the $\mathrm{Ge}_{2} \mathrm{Sb}_{2} \mathrm{Te}_{5}$ device (Fig. 1f). These data demonstrate that once the Sc content is enriched, the crystallization is drastically suppressed in amorphous $\mathrm{Sc}_{x} \mathrm{Sb}_{2} \mathrm{Te}_{3}$ near room temperature. Does this also implicate remarkably weakened driving forces for crystallization at elevated temperatures, causing a prolonged SET time? To clarify this issue, we performed electrical measurements on $\mathrm{Ge}_{2} \mathrm{Sb}_{2} \mathrm{Te}_{5}$ and $\mathrm{Sc}_{x} \mathrm{Sb}_{2} \mathrm{Te}_{3}$ devices to compare the SET speed.

\section{RESET energy and SET speed of PCRAM devices}

Inside the conventional T-shaped PCRAM device (inset of Fig. 2a), a mushroom-shaped amorphous region can be formed through the RESET operation, i.e., melt quenching of the rocksalt PCM film ( $150 \mathrm{~nm}$ in thickness) immediately above the tungsten BEC. The amorphous mushroom regions of different sizes with various RESET resistances will significantly affect the crystallization process in the SET operation ${ }^{6}$. To accurately measure the speed of each SET operation, we preprogrammed all the devices to a fully RESET state by using a very wide constant electric current pulse of $1000 \mathrm{~ns}$. Decreasing the BEC diameter $(\phi)$ from 190 (130) to $80 \mathrm{~nm}$ markedly reduces the RESET energy of the $\mathrm{Sc}_{x} \mathrm{Sb}_{2} \mathrm{Te}_{3}$ and $\mathrm{Ge}_{2} \mathrm{Sb}_{2} \mathrm{Te}_{5}$ devices (Fig. 2a and Figure S1 in the Supplementary information). In particular, the $\mathrm{Sc}_{x} \mathrm{Sb}_{2} \mathrm{Te}_{3}$ devices possess $\sim 85-90 \%$ lower RESET energy (0.64-0.48 $\mathrm{nJ}$ ) than the $\mathrm{Ge}_{2} \mathrm{Sb}_{2} \mathrm{Te}_{5}$ device (4.20 nJ) with a $\phi$ of $80 \mathrm{~nm}$. Such a dramatic reduction in power consumption should be ascribed to the easier melting of the rocksalt lattice of $\mathrm{Sc}_{x} \mathrm{Sb}_{2} \mathrm{Te}_{3}$ that contains a higher concentration of cationic vacancies than $\mathrm{Ge}_{2} \mathrm{Sb}_{2} \mathrm{Te}_{5}{ }^{11,29}$. Upon melting of the rocksalt $\mathrm{Sc}_{\mathrm{x}} \mathrm{Sb}_{2} \mathrm{Te}_{3}$, as revealed by DFMD simulations (Fig. 2b), the central Sc-Te-based cubic motifs remain ordered throughout the heating procedure, whereas the surrounding $\mathrm{Sb}-\mathrm{Te}$ parts can already be fully liquefied. The Sc-Te motif with a strong bond strength promotes disordering of the adjacent $\mathrm{Sb}-\mathrm{Te}$ lattices ${ }^{30}$, through which increasing the Sc content helps lower the RESET energy, although the change is not strikingly large. This also suggests that a slight doping will not significantly change the melting temperature $\left(T_{\mathrm{m}}\right)$ of rocksalt $\mathrm{Sc}_{\mathrm{x}} \mathrm{Sb}_{2} \mathrm{Te}_{3}$. However, it can still essentially alter the crystallization (including both nucleation and growth) behavior $^{23}$. The DFMD simulation here qualitatively illustrates that numerous subcritical $\mathrm{Sc}-\mathrm{Te}$ nucleation embryos can survive the melt-quenching process, along with the quenched-in nuclei of larger size, acting as intrinsic (robust) seeds ${ }^{11,30}$ to speed up the nucleationdominant crystallization (inset of Fig. 2c) in amorphous $\mathrm{Sc}_{x} \mathrm{Sb}_{2} \mathrm{Te}_{3}$.

Voltage pulses of a fixed magnitude but increasing width were imposed on $\mathrm{Ge}_{2} \mathrm{Sb}_{2} \mathrm{Te}_{5}$ and $\mathrm{Sc}_{x} \mathrm{Sb}_{2} \mathrm{Te}_{3}$ devices to assess the SET time (Figure S2 in the Supplementary information). As the magnitude of the applied pulses increases, the SET time can be accordingly shortened (Fig. 2c). We need to note that for the convenience of device fabrication and electrical measurements, we did not 
A

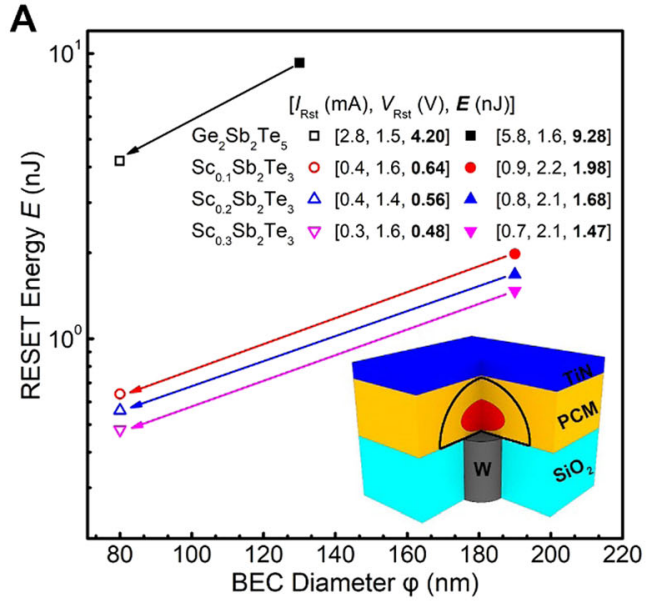

C

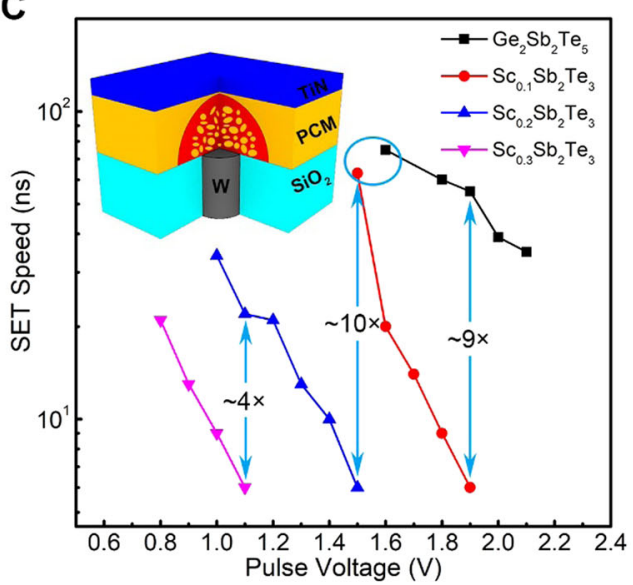

B

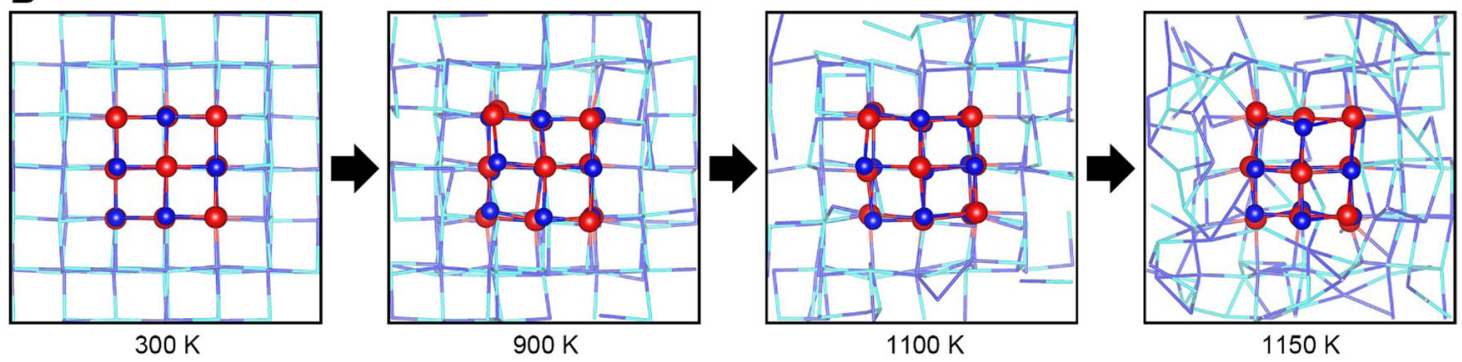

Fig. 2 RESET energy and SET speed of PCRAM devices. a RESET energy $(E)$ as a function of the bottom electrode contact (BEC) diameter $(\varphi)$ for $\mathrm{Ge}_{2} \mathrm{Sb}_{2} \mathrm{Te}_{5}$ and $\mathrm{Sc}_{x} \mathrm{Sb}_{2} \mathrm{Te}_{3}$ devices. The inset shows a schematic of conventional T-shaped PCRAM devices with the following geometry: 150 nmthick PCM layer, $\sim 20$-nm-thick TiN top electrode, and $\sim 80-190 \mathrm{~nm}$ in diameter W bottom electrode. The rocksalt PCM (yellow) immediately above the $\mathrm{BEC}$ is undergoing a melt-quenching process, where the red area represents the supercooled liquid phase. A fully RESET state refers to a large amorphous phase mushroom, as marked by the black contour. The transient RESET voltage $\left(V_{\text {Rst }}\right)$ across the device is recorded once the RESET state is reached. The input RESET energy is calculated as $E=l_{\text {Rst }} \times V_{\text {Rst }} \times t$, where $I_{\text {Rst }}$ is the RESET current, $V_{\text {Rst }}$ is the RESET voltage, and $t$ is fixed at $1000 \mathrm{~ns}$. $\mathbf{b}$ DFMD simulations of the melting process of rocksalt $\mathrm{Sc}_{x} \mathrm{Sb}_{2} \mathrm{Te}_{3}$ showing that the initial structure is still maintained at $900 \mathrm{~K}$ and that the peripheral $\mathrm{Sb}_{2} \mathrm{Te}_{3}$ lattice begins disordering at $1100 \mathrm{~K}$. The surrounding $\mathrm{Sb}_{2} \mathrm{Te}_{3}$ part becomes rather disordered at $1150 \mathrm{~K}$ after $15 \mathrm{ps}$, while the central Sc-Te precursor (Sc: red, Te blue), throughout the whole annealing process, remains in the cubic configuration. c SET operation speed for $\mathrm{Ge}_{2} \mathrm{Sb}_{2} \mathrm{Te}_{5}$ and $\mathrm{Sc}_{x} \mathrm{Sb}_{2} \mathrm{Te}_{3}$ devices with the same geometry. (Inset) Inside the mushroom of the supercooled liquid phase (red), small nuclei/crystallites (yellow) are generated before subsequent crystal growth. Upon the SET operation, all the PCMs show nucleation-dominated crystallization behavior.

extend the testing range to the subnanosecond level; however, the current comparison already suffices to distinguish the differences in the SET speed among the devices. In the $\mathrm{Ge}_{2} \mathrm{Sb}_{2} \mathrm{Te}_{5}$ device, before the largest resistance drop to reach the SET state, the RESET state takes $\sim 20-50$ ns to descend sluggishly to an intermediate (partial) state; in contrast, the resistance drop in all the $\mathrm{Sc}_{x} \mathrm{Sb}_{2} \mathrm{Te}_{3}$ devices is relatively steep (Fig. S2 in the Supplementary information), denoting a faster crystallization process. In Fig. 2c, at a low bias of $\sim 1.5-1.6 \mathrm{~V}$, the $\mathrm{Sc}_{0.1} \mathrm{Sb}_{2} \mathrm{Te}_{3}$ and $\mathrm{Ge}_{2} \mathrm{Sb}_{2} \mathrm{Te}_{5}$ devices have an approximate SET speed of $\sim 70-80 \mathrm{~ns}$; however, at $\sim 1.9 \mathrm{~V}$, the former needs only $\sim 6 \mathrm{~ns}$ to reach SET, whereas the latter still requires $\sim 55 \mathrm{~ns}$. The $\mathrm{Sc}_{0.1} \mathrm{Sb}_{2} \mathrm{Te}_{3}$ device is $\sim 9$ times faster than the $\mathrm{Ge}_{2} \mathrm{Sb}_{2} \mathrm{Te}_{5}$ device. Similarly, at $\sim 1.5 \mathrm{~V}$, the $\mathrm{Sc}_{0.2} \mathrm{Sb}_{2} \mathrm{Te}_{3}$ device ( $\left.\sim \mathrm{ns}\right)$ is over one order of magnitude faster than the $\mathrm{Sc}_{0.1} \mathrm{Sb}_{2} \mathrm{Te}_{3}$ device ( $\left.62 \mathrm{~ns}\right)$. Regarding the
$\mathrm{Sc}_{0.3} \mathrm{Sb}_{2} \mathrm{Te}_{3}$ device $(\sim 6 \mathrm{~ns})$ at $\sim 1.1 \mathrm{~V}$, it is almost four times faster than the $\mathrm{Sc}_{0.2} \mathrm{Sb}_{2} \mathrm{Te}_{3}$ device ( $\left.23 \mathrm{~ns}\right)$. Clearly, enriching the Sc content to $x=0.3$ does not seem to hinder the speed of the SET operation. Note that the SET times of all the $\mathrm{Sc}_{x} \mathrm{Sb}_{2} \mathrm{Te}_{3}$ devices only reach $6 \mathrm{~ns}$ because the minimum pulse width employed here is $6 \mathrm{~ns}$. A further reduction in the SET time for the $\mathrm{Sc}_{0.3} \mathrm{Sb}_{2} \mathrm{Te}_{3}$ device can be expected if picosecond-pulse programming is applied. Although the kinetics near room temperature are largely arrested in $\mathrm{Sc}_{0.3} \mathrm{Sb}_{2} \mathrm{Te}_{3}$, the driving forces for crystallization at elevated temperatures must not have been essentially attenuated; otherwise, the SET speed of the device would be slower than those of the Sc-deficient devices. To lend credence to this hypothesis, we performed FDSC measurements on $\mathrm{Sc}_{x} \mathrm{Sb}_{2} \mathrm{Te}_{3}$ thin films to assess the crystallization kinetics as a function of temperature. 

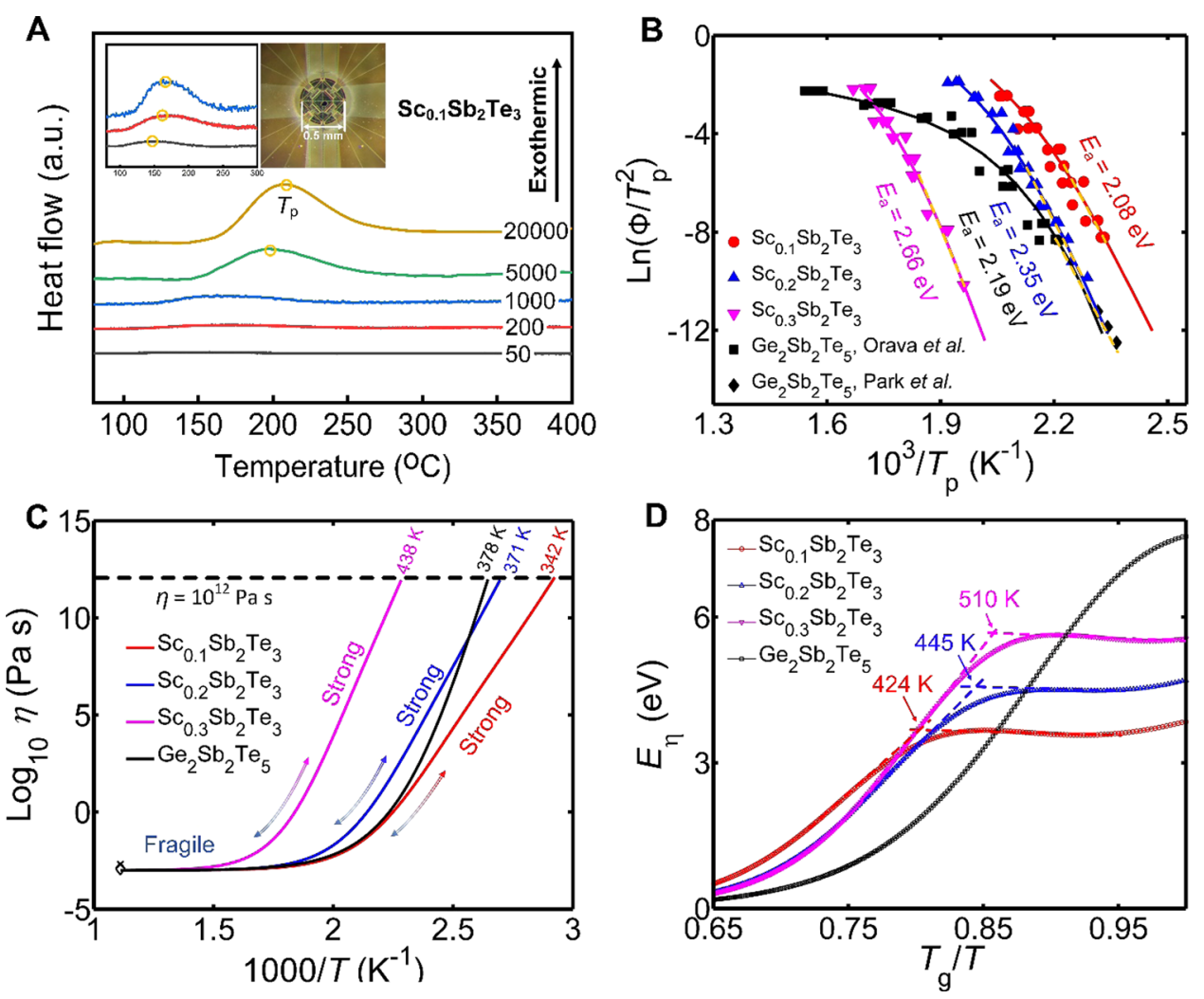

Fig. 3 Enlarged contrast in crystallization kinetics. a Representative FDSC traces of $\mathrm{Sc}_{0.1} \mathrm{Sb}_{2} \mathrm{Te}_{3}$ thin films with $\Phi$ ranging from 50 to $20,000 \mathrm{~K} \mathrm{~s}^{-1}$. The left inset shows a zoom-in view of the crystallization peak temperature $\left(T_{\mathrm{p}}\right)$ at low $\Phi$. The right inset shows the FDSC chip sensor employed in this study. $\mathbf{b}$ Kissinger plots showing the FDSC data of $\mathrm{Sc}_{x} \mathrm{Sb}_{2} \mathrm{Te}_{3}$ and $\mathrm{Ge}_{2} \mathrm{Sb}_{2} \mathrm{Te}_{5}$ (obtained by Orava et al.) ${ }^{13}$ thin films, as well as several data points derived from conventional DSC for $\mathrm{Ge}_{2} \mathrm{Sb}_{2} \mathrm{Te}_{5}$ (obtained by Park et al.). ${ }^{27}$ samples. The crystallization activation energy $E_{\mathrm{a}}$ for $\mathrm{Sc}_{x} \mathrm{Sb}_{2} \mathrm{Te}_{3}$ and $\mathrm{Ge}_{2} \mathrm{Sb}_{2} \mathrm{Te}_{5}$ is derived via linear fitting of the respective data in the lower $\Phi$ range, as marked by the yellow dashed line. $\mathbf{c}$ Temperature-dependent viscosity $\eta$ of $\mathrm{Sc}_{x} \mathrm{Sb}_{2} \mathrm{Te}_{3}$ and $\mathrm{Ge}_{2} \mathrm{Sb}_{2} \mathrm{Te}_{5}$ films. The $T_{\mathrm{g}}$ of each film is set as the temperature where $\eta$ is $10^{12} \mathrm{~Pa}$ s. The $\eta$ of $\mathrm{Ge}_{2} \mathrm{Sb}_{2} \mathrm{Te}_{5}$ at $T_{\mathrm{m}}$ is also plotted in the figure, with the cross $(\mathbf{x})$ indicating the viscosity data measured by an oscillating-cup viscometer ${ }^{32}$ and the open diamond $(\boldsymbol{\nabla})$ the simulated viscosity data ${ }^{19}$, showing good agreement with our results. $\mathbf{d}$ The viscosity activation energy $E_{n}$ for $\mathrm{Sc}_{x} \mathrm{Sb}_{2} \mathrm{Te}_{3}$ reaches a plateau when quenched toward $T_{\mathrm{g}}$, while that of $\mathrm{Ge}_{2} \mathrm{Sb}_{2} \mathrm{Te}_{5}$ increases monotonically. The inflection temperature, i.e., $\sim 424, \sim 445$, and $\sim 510 \mathrm{~K}$, is determined as the FTS crossover point of $\mathrm{Sc}_{0.1} \mathrm{Sb}_{2} \mathrm{Te}_{3}, \mathrm{Sc}_{0.2} \mathrm{Sb}_{2} \mathrm{Te}_{3}$, and $\mathrm{Sc}_{0.3} \mathrm{Sb}_{2} \mathrm{Te}_{3}$, respectively.

\section{Enlarged kinetic contrast through a fragile-to-strong crossover}

The chip sensor of the Mettler-Toledo Flash DSC 1 shown in the right inset of Fig. 3a was employed in the present study. It has a square active area with a length of $\sim 0.3 \mathrm{~mm}$ and allows controlled heating and cooling of samples with a typical mass of $\sim 100 \mathrm{ng}$ up to tens of thousands Kelvin per second ${ }^{13}$. The as-deposited amorphous $\mathrm{Sc}_{x} \mathrm{Sb}_{2} \mathrm{Te}_{3}$ films of $\sim 300 \mathrm{~nm}$ thickness were crystallized by varying the heating rate $(\Phi)$ from 10 to the maximum of $40,000 \mathrm{~K} \mathrm{~s}^{-1}$. Several representative FDSC traces of the $\mathrm{Sc}_{0.1} \mathrm{Sb}_{2} \mathrm{Te}_{3}$ sample after background subtraction are shown in Fig. 3a, where the crystallization peak temperature $\left(T_{\mathrm{p}}\right.$, circled) shifts from $\sim 148$ to $\sim 206^{\circ} \mathrm{C}$ upon increasing $\Phi$ from 50 to $20,000 \mathrm{~K} \mathrm{~s}^{-1}$. The shift of $T_{\mathrm{p}}$ allows for the investigation of the crystallization kinetics at elevated temperatures. Note that the lowest 2 to $3 T_{\mathrm{p}}$ values at each $\Phi$ were regarded as the most credible data because they correspond to the best thermal contact between the stripped-off $\mathrm{Sc}_{x} \mathrm{Sb}_{2} \mathrm{Te}_{3}$ films and chip sensors ${ }^{14,23}$. The crystallization data of $\mathrm{Sc}_{x} \mathrm{Sb}_{2} \mathrm{Te}_{3}$ obtained via FDSC measurements are portrayed in a Kissinger plot (Fig. 3b). Previous $\mathrm{Ge}_{2} \mathrm{Sb}_{2} \mathrm{Te}_{5}$ data obtained by FDSC ${ }^{13}$ and conventional DSC $^{31}$ are also included for a direct comparison. All the $\mathrm{Sc}_{x} \mathrm{Sb}_{2} \mathrm{Te}_{3}$ films obey a linear relation across a wide $\Phi$ range in the Kissinger plot, complying with the Arrhenius behavior up to $\Phi \sim 1000-10,000 \mathrm{~K} \mathrm{~s}^{-1}$, as illustrated by the yellow dashed lines, above which the Arrhenius behavior breaks down. Within the Arrhenius range, the fitted Kissinger plot gives a slope of $E_{\mathrm{a}} \sim 2.08, \sim 2.35$, and $\sim 2.66 \mathrm{eV}$ for $\mathrm{Sc}_{0.1} \mathrm{Sb}_{2} \mathrm{Te}_{3}$, $\mathrm{Sc}_{0.2} \mathrm{Sb}_{2} \mathrm{Te}_{3}$, and $\mathrm{Sc}_{0.3} \mathrm{Sb}_{2} \mathrm{Te}_{3}$, respectively. The values of $E_{\mathrm{a}}$ are reasonably comparable to those obtained in Fig. 1f. In contrast, $\mathrm{Ge}_{2} \mathrm{Sb}_{2} \mathrm{Te}_{5}$ departs from the Arrhenius behavior (with $E_{\mathrm{a}} \sim 2.19 \mathrm{eV}$ ) at a rather low heating rate $(\Phi$ $\left.\sim 100 \mathrm{~K} \mathrm{~s}^{-1}\right)$. Such a broader Arrhenius behavior of 
$\mathrm{Sc}_{x} \mathrm{Sb}_{2} \mathrm{Te}_{3}$ is analogous to those observed in AgIn-doped $\mathrm{Sb}_{2} \mathrm{Te}$ films ${ }^{17}$ and $\mathrm{Ge}_{2} \mathrm{Sb}_{2} \mathrm{Te}_{5}$ nanoparticles ${ }^{14}$. We thereby adopted the same treatments as those in the literature, i.e., the generalized MYEGA model for viscosity combined with Johnson-Mehl-Avrami-Kolmogorov theory to numerically simulate and fit the FDSC data of $\mathrm{Sc}_{x} \mathrm{Sb}_{2} \mathrm{Te}_{3}$ and $\mathrm{Ge}_{2} \mathrm{Sb}_{2} \mathrm{Te}_{5}$ supercooled liquids (see Supplementary information for details).

Figure 3c depicts the temperature dependency of the viscosity $(\eta)$ determined from the generalized MYEGA model (see Equation S3, Table S1 and discussions in the Supplementary information). The modeled viscosity shows an excellent match with simulated and experimental data at $T_{\mathrm{m}}$ reported previously ${ }^{19,32}$. Note that in the generalized MYEGA model, the glass transition temperature $\left(T_{\mathrm{g}}\right)$ is not directly provided but is set as the temperature where $\eta$ reaches $10^{12} \mathrm{~Pa}$ s. The fitted $T_{\mathrm{g}}$ of $\mathrm{Sc}_{0.1} \mathrm{Sb}_{2} \mathrm{Te}_{3}, \mathrm{Sc}_{0.2} \mathrm{Sb}_{2} \mathrm{Te}_{3}$, and $\mathrm{Sc}_{0.3} \mathrm{Sb}_{2} \mathrm{Te}_{3}$ is 342,371 , and $438 \mathrm{~K}$, respectively, and our $T_{\mathrm{g}}$ of $\mathrm{Ge}_{2} \mathrm{Sb}_{2} \mathrm{Te}_{5}$, i.e., $378 \mathrm{~K}$, is comparable to the previously reported value of $\sim 383 \mathrm{~K}^{13}$. In between $T_{\mathrm{g}}$ and $T_{\mathrm{m}}$, strong liquids such as silica follow the Arrhenius behavior with a nearly constant activation energy for viscous flow, whereas fragile liquids such as oterphenyl $^{33}$ exhibit a rather high activation energy near $T_{\mathrm{g}}$ but become markedly less viscous as the temperature increases. The fragility $m$, defined as the slope of the $\eta$ at $T_{\mathrm{g}}$, i.e., $\mathrm{m}=\mathrm{d}\left[\log _{10} \eta(T)\right] /\left.d\left(T_{g} / T\right)\right|_{T=T g}$, is derived as $\sim 56$, $\sim 64$, and $\sim 64$ for $\mathrm{Sc}_{0.1} \mathrm{Sb}_{2} \mathrm{Te}_{3}, \quad \mathrm{Sc}_{0.2} \mathrm{Sb}_{2} \mathrm{Te}_{3}$, and $\mathrm{Sc}_{0.3} \mathrm{Sb}_{2} \mathrm{Te}_{3}$, respectively. $\mathrm{Ge}_{2} \mathrm{Sb}_{2} \mathrm{Te}_{5}(m \sim 102)$ is apparently more fragile than $\mathrm{Sc}_{x} \mathrm{Sb}_{2} \mathrm{Te}_{3}$ when approaching $T_{\mathrm{g}}$. If a single-fragility model is employed to describe the viscosity behavior of $\mathrm{Sc}_{x} \mathrm{Sb}_{2} \mathrm{Te}_{3}$, then it can only be applicable to the high-temperature regime above $\sim 400-500 \mathrm{~K}$, generating rather large fragilities of over $\sim 170$. Instead, the generalized MYEGA model can nicely fit not only the curved (non-Arrhenius behavior) but also the linear (Arrhenius behavior) parts in the Kissinger plot ${ }^{14,23}$. The high-to-low change in the fragility strongly indicates that an enlarged contrast in the kinetics occurs below and above these inflection temperatures at $\sim 400-500 \mathrm{~K}$, manifesting as an apparent fragile-to-strong (FTS) crossover in the deeply supercooled $\mathrm{Sc}_{x} \mathrm{Sb}_{2} \mathrm{Te}_{3}$ liquid (Fig. 3c, d).

The FTS crossover can be assessed by monitoring $E_{\eta}=$ $k_{B} d \ln \left(\eta / \eta_{0}\right) / d(1 / \mathrm{T})$, where $k_{\mathrm{B}}$ is the Boltzmann constant and $E_{\eta}$ is the viscosity activation energy. $\mathrm{Sc}_{x} \mathrm{Sb}_{2} \mathrm{Te}_{3}$ approximately obeys the Arrhenius behavior along a clear plateau in $E_{\eta}$ from $T_{\mathrm{g}}$ to each inflection point, i.e., $\sim 424$, $\sim 445$, and $\sim 510 \mathrm{~K}$, beyond which $E_{\eta}$ sharply drops, approaching zero at $T_{\mathrm{g}} / T \approx 0.65$, thus presenting the super-Arrhenius behavior of increased fluidity (Fig. 3d). These inflection points can be taken as the FTS temperatures for $\mathrm{Sc}_{x} \mathrm{Sb}_{2} \mathrm{Te}_{3}$. In contrast, our fitting did not identify any clear FTS crossover in $\mathrm{Ge}_{2} \mathrm{Sb}_{2} \mathrm{Te}_{5}$ above its $T_{\mathrm{g}}$, despite a potentially existing crossover at even lower temperatures ${ }^{17}$. The FTS crossover may originate from the increase in the Peierls distortion in the short range and the formation of an energetically favorable network at or beyond the medium range during quenching of the supercooled liquid ${ }^{34-36}$. As the Peierls distortion increases, electrons become more localized between atoms, exerting constraints on atomic migration and consequently increasing the activation barrier for viscous flow. The enhanced kinetic contrast due to the FTS crossover enables high atomic mobility at elevated temperatures for rapid crystal growth in a less-viscous matrix while suppressing atomic diffusion near room temperature for good data retention in a more-viscous state. If the FTS crossover occurs at either below $T_{\mathrm{g}}$ (e.g., in $\left.\mathrm{Ge}_{2} \mathrm{Sb}_{2} \mathrm{Te}_{5}\right)^{17}$ or above $T_{\mathrm{m}}$ (e.g., in $\left.\mathrm{Ge}_{15} \mathrm{Te}_{85}\right)^{37}$, it would cause relatively less- or more-viscous flow with inferior data retention or slower crystal growth, respectively. Regarding this, a desirable FTS crossover for an ultrafast and persistent PCM should occur immediately below the typical programming temperatures for crystallization $(<\sim 600-700 \mathrm{~K})$ and considerably above its $T_{\mathrm{g}}(>\sim 400 \mathrm{~K})$, as exemplified by the cases of $\mathrm{Sc}_{x} \mathrm{Sb}_{2} \mathrm{Te}_{3}(x=\sim 0.1-0.3)$. Note that an increase in the Sc dose produces a more intensive change in the kinetics (with a larger contrast in $E_{\eta}$ ) upon a relatively small temperature range from the close $T_{\mathrm{m}}$ to the higher FTS temperature (Fig. 3d). This will undoubtedly essentially alter not only the amorphous stability when approaching room temperature but also, most importantly, the crystallization speed at elevated temperatures.

\section{Crystal growth velocity and heterogeneous nucleation rate}

Generally, in the crystallization of a glass through continuous heating, the peak in the nucleation rate usually precedes the peak in the growth velocity. Accordingly, in analyzing the FDSC data of PCMs to derive the crystal growth velocity $(U)$, a reasonably simplified method was usually applied by assuming that the nucleation has already finished before the peak growth of a fixed population of small crystallites occurs ${ }^{13,14}$. It is known that this simplification does not significantly alter the kinetics results derived compared to the method adopting a more intricate nucleation model $^{14}$. The temperature-dependent $U$ (Fig. 4a) can then be obtained from Kissinger analysis of the shift of the peak position in Fig. 3b (see Equation S1 and discussions in the Supplementary Information). The maximum growth velocity $\left(U_{\max }\right)$ is $\sim 3.14, \sim 2.07$, and $\sim 1.45 \mathrm{~m} \mathrm{~s}^{-1}$ for $\mathrm{Sc}_{0.1} \mathrm{Sb}_{2} \mathrm{Te}_{3}$ (at $\sim 0.70 T_{\mathrm{m}}$ ), $\mathrm{Sc}_{0.2} \mathrm{Sb}_{2} \mathrm{Te}_{3}$ (at $\sim 0.73 T_{\mathrm{m}}$ ), and $\mathrm{Sc}_{0.3} \mathrm{Sb}_{2} \mathrm{Te}_{3}$ (at $\left.\sim 0.80 T_{\mathrm{m}}\right)$, respectively, where $T_{\mathrm{m}}(\sim 890 \mathrm{~K})$ is assumed to be roughly equal to that of $\mathrm{Sb}_{2} \mathrm{Te}_{3}$. The $U_{\max }(\sim 1.92 \mathrm{~m}$ $\mathrm{s}^{-1}$ ) of $\mathrm{Ge}_{2} \mathrm{Sb}_{2} \mathrm{Te}_{5}$ appears at $\sim 0.70 T_{\mathrm{m}}$, with $T_{\mathrm{m}}$ being $\sim 900 \mathrm{~K}$. Such a $U_{\max }$ for $\mathrm{Ge}_{2} \mathrm{Sb}_{2} \mathrm{Te}_{5}$ well matches previous results measured by FDSC and on real PCRAM devices, ranging from $\sim 0.5$ to $3 \mathrm{~m} \mathrm{~s}^{-1},{ }^{13-15}$. 

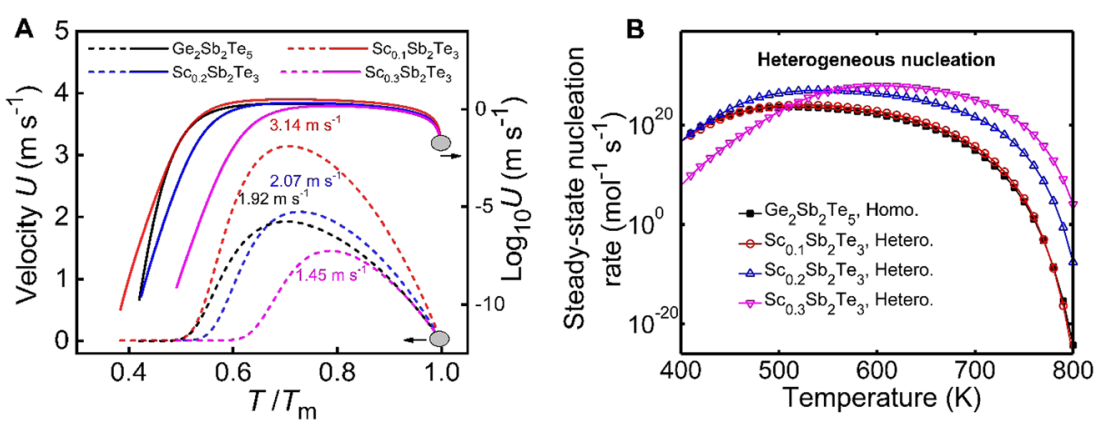

Fig. 4 Crystal growth velocity and nucleation rate. a Crystal growth velocities $U$ of $\mathrm{Sc}_{x} \mathrm{Sb}_{2} \mathrm{Te}_{3}$ and $\mathrm{Ge}_{2} \mathrm{Sb}_{2} \mathrm{Te}_{5}$ supercooled liquids between $T_{\mathrm{g}}$ and $T_{\mathrm{m}}$. The maximum velocity $U_{\max }$ value of each material is also shown near the corresponding peak. As the Sc content increases, the peak becomes shorter and shifts to higher temperatures. b Steady-state nucleation rate $\rho^{55}$ of $\mathrm{Ge}_{2} \mathrm{Sb}_{2} \mathrm{Te}_{5}$ (homogeneous, abbreviated as $\mathrm{Homo}_{\text {.) }}$ and $\mathrm{Sc}_{x} \mathrm{Sb}_{2} \mathrm{Te}_{3}$ (heterogeneous, abbreviated as Hetero.) with a contact angle $\theta$ of $20^{\circ}$ taking the size effect into account.

According to the Stokes-Einstein relation in the Arrhenius case, the atomic diffusivity $D$ is inversely proportional to the viscosity $\eta$, i.e., $U \propto D \propto \eta^{1}$. When this relation breaks down, there will be decoupling between $U$ and $\eta$, resulting in $\mathrm{U} \propto \eta^{-\xi}$ with $\xi<1$, i.e., even faster crystal growth. Via fitting, the $\xi$ for $\mathrm{Sc}_{0.1} \mathrm{Sb}_{2} \mathrm{Te}_{3}$, $\mathrm{Sc}_{0.2} \mathrm{Sb}_{2} \mathrm{Te}_{3}$, and $\mathrm{Sc}_{0.3} \mathrm{Sb}_{2} \mathrm{Te}_{3}$ is determined to be $\sim 0.71$, $\sim 0.65$, and $\sim 0.63$, respectively, and that for $\mathrm{Ge}_{2} \mathrm{Sb}_{2} \mathrm{Te}_{5}$ is $\sim 0.67$. In Fig. $3 \mathrm{c}$, from its $T_{\mathrm{g}}(\sim 438 \mathrm{~K})$ to $\sim 700 \mathrm{~K}$ (i.e., $1,000 / T=\sim 1.43), \mathrm{Sc}_{0.3} \mathrm{Sb}_{2} \mathrm{Te}_{3}$ has a very large $\eta$ many orders of magnitude larger than those of the other materials; unsurprisingly, its $U$ remains the smallest (Fig. 4a). $\mathrm{Sc}_{0.1} \mathrm{Sb}_{2} \mathrm{Te}_{3}$ is the opposite, behaving as the leastviscous liquid from its $T_{\mathrm{g}}(\sim 342 \mathrm{~K})$ to $T_{\mathrm{m}}$, which ensures the largest $U$ (up to $\sim 1.6$ times that of $\mathrm{Ge}_{2} \mathrm{Sb}_{2} \mathrm{Te}_{5}$ ). For $\mathrm{Sc}_{0.2} \mathrm{Sb}_{2} \mathrm{Te}_{3}$, its $\eta$ is also considerably larger (< 100-fold) than that of $\mathrm{Ge}_{2} \mathrm{Sb}_{2} \mathrm{Te}_{5}$ between $\sim 400$ and $570 \mathrm{~K}$ (i.e., $1,000 / T=\sim 1.75-2.50$, in Fig. 3c), corresponding to a markedly slower $U(<\sim 120$-fold) than that of the latter in the $T$ range from $\sim 0.44$ to $\sim 0.64 T_{\mathrm{m}}$ (Fig. 4a). However, beyond $\sim 570 \mathrm{~K}$, due to the almost equal $\eta$ and a slightly smaller $\xi$, the $U$ of $\mathrm{Sc}_{0.2} \mathrm{Sb}_{2} \mathrm{Te}_{3}$ becomes comparable to (i.e., $\sim 1.1$ times larger than) that of $\mathrm{Ge}_{2} \mathrm{Sb}_{2} \mathrm{Te}_{5}$. Obviously, it would be irrational to ascribe the significant differences (over 10 times) in the SET speed only to the crystal growth momentum, especially when the weakest material $\left(\mathrm{Sc}_{0.3} \mathrm{Sb}_{2} \mathrm{Te}_{3}\right)$ becomes the fastest. Nucleation must play a decisive role in accelerating the whole crystallization process.

Previously, we proved that homogeneous nucleation is invalid in interpreting the much faster crystallization nature of $\mathrm{Sc}_{\mathrm{x}} \mathrm{Sb}_{2} \mathrm{Te}_{3}{ }^{23}$. Regarding heterogeneous nucleation, if we choose a fairly small contact angle $\theta$ (say less than $30^{\circ}$, resembling the situation in supercooled water) ${ }^{38}$, $\mathrm{Sc}_{0.3} \mathrm{Sb}_{2} \mathrm{Te}_{3}$, strangely, has an extremely lower heterogeneous steady-state nucleation rate $\left(I_{\text {het }}^{\text {ss }}\right)$ than the other two materials below $\sim 630 \mathrm{~K}$, which just slightly surpasses their rates at higher temperatures (Fig. S3 in the
Supplementary information). Note that below $\sim 630 \mathrm{~K}$, $\mathrm{Sc}_{0.3} \mathrm{Sb}_{2} \mathrm{Te}_{3}$ possesses approximately twofold to to approximately seven orders of magnitude lower $U$ than the other two materials (Fig. 4a). Combining these two characteristics, a contradictory scenario against Fig. 2c is depicted in that $\mathrm{Sc}_{0.3} \mathrm{Sb}_{2} \mathrm{Te}_{3}$ should crystallize much slower in the device than the other materials. We thus modified the common description of heterogeneous nucleation by taking the size effect of the nucleation precursor $^{38}$ into consideration (see Equations S14-S19, Table S2 and discussions in the Supplementary information).

Figure $4 \mathrm{~b}$ shows the comparison among the homogeneous steady-state nucleation rate $\left(I_{\text {hom }}^{\mathrm{ss}}\right)$ of $\mathrm{Ge}_{2} \mathrm{Sb}_{2} \mathrm{Te}_{5}$ and the modified $I_{\text {het }}^{\mathrm{ss}}$ of $\mathrm{Sc}_{x} \mathrm{Sb}_{2} \mathrm{Te}_{3}$. Although very subtle, it can be determined that the $I_{\text {het }}^{S S}$ of $\mathrm{Sc}_{0.1} \mathrm{Sb}_{2} \mathrm{Te}_{3}$ is actually slightly larger than the $I_{\text {hom }}^{\text {ss }}$ of $\mathrm{Ge}_{2} \mathrm{Sb}_{2} \mathrm{Te}_{5}$, e.g., $\sim 2-6$-fold at $\sim 500-700 \mathrm{~K}$, and in this range, the former also has an up to $\sim 1.5$ times higher $U$ than the latter (Fig. 4a). In this way, the $\mathrm{Sc}_{0.1} \mathrm{Sb}_{2} \mathrm{Te}_{3}$ device can be $\sim 9$ times faster than the $\mathrm{Ge}_{2} \mathrm{Sb}_{2} \mathrm{Te}_{5}$ device in the SET operation. In contrast, it is easier to distinguish that the $I_{\text {het }}^{s \mathrm{~s}}$ of $\mathrm{Sc}_{0.2} \mathrm{Sb}_{2} \mathrm{Te}_{3}$ is constantly larger, i.e., by $\sim 2-5$ orders of magnitude, than that of $\mathrm{Sc}_{0.1} \mathrm{Sb}_{2} \mathrm{Te}_{3}$ in the regime of $\sim 500-700 \mathrm{~K}$. Despite the relatively low growth velocity, $\mathrm{Sc}_{0.2} \mathrm{Sb}_{2} \mathrm{Te}_{3}$ easily outperforms $\mathrm{Sc}_{0.1} \mathrm{Sb}_{2} \mathrm{Te}_{3}$ in SET speed owing to the plenty of robust $\mathrm{Sc}-\mathrm{Te}$ nuclei compared to the latter. The most interesting message here is from $\mathrm{Sc}_{0.3} \mathrm{Sb}_{2} \mathrm{Te}_{3}$ : only beyond $\sim 550 \mathrm{~K}$ can its $I_{\text {het }}^{\text {ss }}$ exceed that of $\mathrm{Sc}_{0.2} \mathrm{Sb}_{2} \mathrm{Te}_{3}$, but the latter decays noticeably faster than the former, where the difference enlarges steadily from approximately tenfold at $\sim 600 \mathrm{~K}$ to $\sim 3-5$ orders of magnitude at $\sim 700-750 \mathrm{~K}$. In light of the merely $<\sim 4$ times slower $U$ at $\sim 600-700 \mathrm{~K}$ (i.e., $\quad T / T_{\mathrm{m}}=\sim 0.67-0.79$ in Fig. $4 \mathrm{a}$ ), $\quad \mathrm{Sc}_{0.3} \mathrm{Sb}_{2} \mathrm{Te}_{3}$ undoubtedly can crystallize much faster than $\mathrm{Sc}_{0.2} \mathrm{Sb}_{2} \mathrm{Te}_{3}$ in the SET operation.

In a very-viscous supercooled liquid near above $T_{\mathrm{g}}$, nucleation becomes rather difficult ${ }^{17,39}$. This is the case 
here for $\mathrm{Sc}_{0.3} \mathrm{Sb}_{2} \mathrm{Te}_{3}$, whose viscosity below the FTS temperature of $\sim 510 \mathrm{~K}$ is very large with respect to the others (Fig. 3c). However, on the other hand, enriching the Sc content is helpful for increasing the number or size of the Sc-Te-based nucleation precursors, by which the heterogeneous nuclei can be more robust (of reduced stochasticity), withstanding higher-temperature fluctuations. However, one cannot arbitrarily use heavy Sc doping to pursue a very high nucleation rate ${ }^{40}$ because the atomic mobility throughout a wide supercooled liquid regime would be considerably suppressed, especially within the typical SET programming window of $\sim 600-700 \mathrm{~K}$. Thereby, the growth velocity would be severely reduced, and both the nucleation rate and growth velocity peaks would be shifted to an even highertemperature zone. Since the driving forces for crystallization weaken as the temperature approaches $T_{\mathrm{m}}{ }^{1,2}$, the nucleation and subsequent growth would strongly decay to rather low levels, leaving a quite narrow programming window for achieving a fast SET speed. To support this point, we further checked the FDSC data of $\mathrm{Sc}_{0.36} \mathrm{Sb}_{2} \mathrm{Te}_{3}$ (Fig. S4 in the Supplementary information). It is certainly a more viscous liquid than $\mathrm{Sc}_{0.3} \mathrm{Sb}_{2} \mathrm{Te}_{3}$ in the deeply supercooled regime, due to which its crystal growth is dramatically restrained with a peak value of only $\sim 0.75 \mathrm{~m}$ $\mathrm{s}^{-1}$ postponed to $\sim 735 \mathrm{~K}$ (at $\sim 0.83 T_{\mathrm{m}}$ ), while preceding major growth, its nucleation rate is approximately the same as or even significantly lower than that of $\mathrm{Sc}_{0.3} \mathrm{Sb}_{2} \mathrm{Te}_{3}$. As the temperature further increases to $\sim 800 \mathrm{~K}$ (at $\sim 0.90 T_{\mathrm{m}}$ ), both the nucleation tendency and growth momentum are sharply weakened. Clearly, $\mathrm{Sc}_{0.36} \mathrm{Sb}_{2} \mathrm{Te}_{3}$ has little chance to outperform $\mathrm{Sc}_{0.3} \mathrm{Sb}_{2} \mathrm{Te}_{3}$ in crystallization speed.

\section{Conclusions}

Faster crystallization speed and better amorphous stability are the two 'conflicting' properties in pursuing ultrafast and persistent phase-change working memory. $\mathrm{Sc}_{x} \mathrm{Sb}_{2} \mathrm{Te}_{3}$ is the most promising PCM system for reconciling the contradiction. We proved that as the $\mathrm{Sc}$ content increases from $x=0.1-0.3$ in the $\mathrm{Sc}_{x} \mathrm{Sb}_{2} \mathrm{Te}_{3}$ device, the data retention ability of the RESET state can be monotonically enhanced, whereas the SET operation also becomes faster accordingly. We thus performed FDSC experiments to unravel the seemingly strange temperature-dependent crystallization dynamics in the supercooled liquid regime. We found that enriching Sc increases the viscosity of the supercooled liquid and shapes broader Arrhenius-like kinetics from $T_{\mathrm{g}}$ up to an apparent FTS crossover. The rather suppressed atomic diffusion in the 'strong' liquid greatly restrains the crystallization near room temperature, strengthening the amorphous phase to provide long-term nonvolatility, while above the inflection point in the kinetics, the 'strong' liquid crossovers into a 'fragile' liquid of lessviscous flow, allowing high atomic mobility for fast crystal growth at elevated temperatures. The maximum crystal growth velocity of $\mathrm{Sc}_{x} \mathrm{Sb}_{2} \mathrm{Te}_{3} \quad(x=0.1-0.3)$ is $\sim 3.14-1.45 \mathrm{~m} \mathrm{~s}^{-1}$, occurring at $\sim 623-712 \mathrm{~K}$, which is more or less comparable to that of $\mathrm{Ge}_{2} \mathrm{Sb}_{2} \mathrm{Te}_{5}(\sim 1.92 \mathrm{~m}$ $\mathrm{s}^{-1}$ at $\left.\sim 630 \mathrm{~K}\right)$. Nonetheless, in the typical programming temperature range for crystallization, i.e., $\sim 600-700 \mathrm{~K}$, the heterogeneous nucleation rate of $\mathrm{Sc}_{x} \mathrm{Sb}_{2} \mathrm{Te}_{3}(x=0.1-0.3)$ overwhelms the homogeneous nucleation rate in $\mathrm{Ge}_{2} \mathrm{Sb}_{2} \mathrm{Te}_{5}$ by several fold to many orders of magnitude; therefore, one can witness a hierarchy in the SET speed from the slowest $\mathrm{Ge}_{2} \mathrm{Sb}_{2} \mathrm{Te}_{5}$ to the fastest $\mathrm{Sc}_{0.3} \mathrm{Sb}_{2} \mathrm{Te}_{3}$ device. Note, however, that doping extra Sc over $\mathrm{Sc}_{0.3} \mathrm{Sb}_{2} \mathrm{Te}_{3}$ may have a negative impact on the crystallization speed because the driving forces would be substantially suppressed below $\sim 735 \mathrm{~K}$. Overall, our work illustrates a route to design superior PCMs for the development of phase-change working memory; that is, while promoting the nucleation rate at elevated temperatures, the kinetic contrast between elevated and ambient temperatures should be enlarged in a cautious manner, preventing the high-temperature crystal growth velocity from seriously decreasing.

\section{Acknowledgements \\ F.R. gratefully thanks the Major Provincial Basic Research Program of Guangdong (2017KZDXM070) and the Science and Technology Foundation of Shenzhen (JCYJ20180507182248605) for the support. K.D. thanks the Science and Technology Foundation of Shenzhen (JCYJ20190808150605474) for the support. Y.C. thanks the National Natural Science Foundation of China (61904091) for the support. J.W. thanks the National Natural Science Foundation of China (51771216) for the support. X.S. thanks the National Natural Science Foundation of China (61775111) for the support. The supercomputer time was provided by the National Supercomputer Center in Tianjin, and the calculations were performed on TianHe-1 (A).}

\section{Author details}

${ }^{1}$ College of Materials Science and Engineering, Shenzhen University, 518060 Shenzhen, China. ${ }^{2}$ Key Laboratory of Optoelectronic Devices and Systems of Ministry of Education and Guangdong Province, College of Optoelectronic Engineering, Shenzhen University, 518060 Shenzhen, China. ${ }^{3}$ Department of Microelectronic Science and Engineering, School of Physical Science and Technology, Ningbo University, 315211 Ningbo, China. ${ }^{4}$ Laboratory of Infrared Material and Devices \& Key Laboratory of Photoelectric Materials and Devices of Zhejiang Province, Advanced Technology Research Institute, Ningbo University, 315211 Ningbo, China. ${ }^{5}$ CAS Key Laboratory of Magnetic Materials and Devices \& Zhejiang Province Key Laboratory of Magnetic Materials and Application Technology, Ningbo Institute of Materials Technology \& Engineering, Chinese Academy of Sciences, 315201 Ningbo, China

\section{Author contributions}

K.D. and B.C. fabricated the PCM film samples. K.D. carried out sheet resistance characterizations and TEM experiments. KD. and F.R. prepared the device samples and carried out electrical measurements. Y.C. performed FDSC measurements. B.C. performed simulation and fitting of FDSC data. F.R., B.C., and K.D. carried out theoretical analysis and together wrote this paper with help from Y.C., J.W., and X.S. The project was initiated and conceptualized by F.R.

Conflict of interest

The authors declare that they have no conflict of interest. 


\section{Publisher's note}

Springer Nature remains neutral with regard to jurisdictional claims in published maps and institutional affiliations.

Supplementary information is available for this paper at https://doi.org/ 10.1038/s41427-020-00246-z.

Received: 3 May 2020 Revised: 10 July 2020 Accepted: 17 July 2020. Published online: 25 September 2020

\section{References}

1. Wuttig, M. \& Yamada, N. Phase-change materials for rewriteable data storage. Nat. Mater. 6, 824-832 (2007).

2. Raoux, S., Welnic, W. \& lelmini, D. Phase change materials and their application to nonvolatile memories. Chem. Rev. 110, 240-267 (2010).

3. Kolobov et al. Understanding the phase-change mechanism of rewritable optical media. Nat. Mater. 3, 703-708 (2004).

4. Atwood, G. Phase-change materials for electronic memories. Science 321, 210-211 (2008)

5. Ielmini, D. \& Lacaita, A. L. Phase change materials in non-volatile storage. Mater. Today 14, 600-607 (2011).

6. Ding, K. Y. et al. Phase-change heterostructure enables ultralow noise and drift for memory operation. Science 366, 210-215 (2019).

7. Wong, H. S. \& Salahuddin, S. Memory leads the way to better computing. Nat. Nanotechnol. 10, 191-194 (2015).

8. Choe, J. Tech insights: Intel 3D XPoint memory die removed from Intel Optane PCM (phase change memory), https://www.techinsights.com/blog/ intel-3d-xpoint-memory-die-removed-inteloptanetm-pcm-phase-changememory.

9. Loke, D. et al. Breaking the speed limits of phase-change memory. Science 336, 1566-1569 (2012).

10. Rao, F. et al. Direct observation of titanium-centered octahedra in titanium-antimony-tellurium phase-change material. Nat. Commun. 6, 10040 (2015).

11. Rao, F. et al. Reducing the stochasticity of crystal nucleation to enable subnanosecond memory writing. Science 358, 1423-1427 (2017).

12. Hegedüs, J. \& Elliott, S. R. Microscopic origin of the fast crystallization ability of Ge-Sb-Te phase-change memory materials. Nat. Mater. 7, 399-405 (2008).

13. Orava, J., Greer, A. L., Gholipour, B., Hewak, D. W. \& Smith, C. E. Characterization of supercooled liquid $\mathrm{Ge}_{2} \mathrm{Sb}_{2} \mathrm{Te}_{5}$ and its crystallization by ultrafast-heating calorimetry. Nat. Mater. 11, 279-283 (2012).

14. Chen, B., Brink, G. H., Palasantzas, G. \& Kooi, B. J. Crystallization kinetics of GeSbTe phase-change nanoparticles resolved by ultrafast calorimetry. J. Phys. Chem. C. 121, 8569-8578 (2017).

15. Sebastian, A., Le Gallo, M. \& Krebs, D. Crystal growth within a phase change memory cell. Nat. Commun. 5, 4314 (2014).

16. Kim, I. S. et al. High performance PRAM cell scalable to sub-20 nm technology with below $4 \mathrm{~F}^{2}$ cell size, extendable to DRAM applications. Symposium on VLSI Technology, 203-204 (2010).

17. Orava, J., Hewak, D. W. \& Greer, A. L. Fragile-to-strong crossover in supercooled liquid Ag-In-Sb-Te studied by ultrafast calorimetry. Adv. Funct. Mater. 25, 4851-4858 (2015).

18. Kalikka, J., Akola, J. \& Jones, R. O. Crystallization processes in the phase change material $\mathrm{Ge}_{2} \mathrm{Sb}_{2} \mathrm{Te}_{5}$ : Unbiased density functional/molecular dynamics simulations. Phys. Rev. B 94, 134105 (2016).
19. Akola, J. \& Jones, R. O. Structural phase transitions on the nanoscale: The crucial pattern in the phase-change materials $\mathrm{Ge}_{2} \mathrm{Sb}_{2} \mathrm{Te}_{5}$ and GeTe. Phys. Rev. B 76, 23501 (2007).

20. Navarro, G. et al. Trade-off between SET and data retention performance thanks to innovative materials for phase-change memory. IEEE International Electron Devices Meeting (IEDM), 570-573 (2013).

21. Cheng, H. Y. et al. A high performance phase change memory with fast switching speed and high temperature retention by engineering the $\mathrm{Ge}_{x} \mathrm{~S}-$ $\mathrm{b}_{y} \mathrm{Te}_{z}$ phase change material. IEEE International Electron Devices Meeting (IEDM), 51-54 (2011).

22. Zhou, X. L. et al. Understanding phase-change behaviors of carbon-doped $\mathrm{Ge}_{2} \mathrm{Sb}_{2} \mathrm{Te}_{5}$ for phase-change memory application. ACS Appl. Mater. Interfaces $\mathbf{6}$, 14207-14214 (2014)

23. Chen, B. et al. Kinetics features conducive to cache-type nonvolatile phasechange memory. Chem. Mater. 31, 8794-8800 (2019).

24. Chen, B., de Wal, D., ten Brink, G. H., Palasantzas, G. \& Kooi, B. J. Resolving crystallization kinetics of GeTe phase-change nanoparticles by ultrafast calorimetry. Cryst. Growth Des. 18, 1041-1046 (2018).

25. Kresse, G. \& Hafner, J. Ab initio molecular dynamics for liquid metals. Phys. Rev. B 47, 558-561 (1993).

26. Perdew, J. P., Burke, K. \& Ernzerhof, M. Generalized gradient approximation made simple. Phys. Rev. Lett. 77, 3865-3868 (1996).

27. Blöchl, P. E. Projector augmented-wave method. Phys. Rev. B 50, 17953-17979 (1994).

28. Rao, F. et al. Investigation of changes in band gap and density of localized states on phase transition for $\mathrm{Ge}_{2} \mathrm{Sb}_{2} \mathrm{Te}_{5}$ and $\mathrm{Si}_{3} \mathrm{Sb}_{2} \mathrm{Te}_{3}$ materials. Acta Mater. 60, 323-328 (2012)

29. Zheng, Y. et al. Direct observation of metastable face-centered cubic $\mathrm{Sb}_{2} \mathrm{Te}_{3}$ crystal. Nano Res 9, 3453-3462 (2016).

30. Wang, X. P. et al. Time-dependent density-functional theory moleculardynamics study on amorphization of Sc-Sb-Te alloy under optical excitation. NPJ Comput. Mater. 6, 31 (2020).

31. Park, J., Kim, M. R., Choi, W. S., Seo, H. \& Yeon, C. Characterization of amorphous phases of $\mathrm{Ge}_{2} \mathrm{Sb}_{2} \mathrm{Te}_{5}$ phase-change optical recording material on their crystallization behavior. Jpn. J. Appl. Phys. 38, 4775-4779 (1999).

32. Schumacher, M. et al. Structural, electronic and kinetic properties of the phase-change material $\mathrm{Ge}_{2} \mathrm{Sb}_{2} \mathrm{Te}_{5}$ in the liquid state. Sci. Rep. 6, 27434 (2016).

33. Angell, C. A. Formation of glasses from liquids and biopolymers. Science $\mathbf{2 6 7}$, 1924-1935 (1995).

34. Rao, F., Zhang, W. \& Ma, E. Catching structural transition in liquids. Science $\mathbf{3 6 4}$, 1032-1033 (2019).

35. Zalden, P. et al. Femtosecond $X$-ray diffraction reveals a liquid-liquid phase transition in phase-change materials. Science 364, 1062-1067 (2019).

36. Sun, Z. M., Zhou, J., Mao, H. K. \& Ahuja, R. Peierls distortion mediated reversible phase transition in GeTe under pressure. Proc Natl Acad. Sci. USA 109, 5948-5952 (2012)

37. Weber, H., Orava, J., Kaban, I., Pries, J. \& Greer, A. L. Correlating ultrafast calorimetry, viscosity, and structural measurements in liquid GeTe and $\mathrm{Ge}_{15} \mathrm{Te}_{85}$. Phys. Rev. Mater. 2, 093405 (2018).

38. Fletcher, N. H. Size effect in heterogeneous nucleation. J. Chem. Phys. 29 572-576 (1958).

39. Orava, J. \& Greer, A. L. Classical-nucleation-theory analysis of priming in chalcogenide phase-change memory. Acta Mater. 139, 226-235 (2017).

40. Zewdie, G. M. et al. Chemical design principles for cache-type Sc-Sb-Te phasechange memory materials. Chem. Mater. 31, 4008-4015 (2019). 